\title{
RULING OUT MULTIPLICITY AND INDETERMINACY: THE ROLE OF HETEROGENEITY *
}

\author{
Berthold Herrendorf ${ }^{1}$, Ákos Valentinyi ${ }^{2}$ and Robert Waldmann ${ }^{3}$
}

\begin{abstract}
It is well known that economies of scale that are external to the individual decision makers can lead to self-fulfilling prophecies and the multiplicity or even indeterminacy of equilibrium. We argue that the importance of this source of multiplicity and indeterminacy is overstated in representative agent models, as they ignore the potential stabilizing effect of heterogeneity. We illustrate this point in a version of Matsuyama`s (1991) two-sector model with increasing returns to scale. Two main results are shown. First, sufficient homogeneity with respect to individual productivity leads to the instability and non-uniqueness of a given stationary state and the indeterminacy of equilibrium at that stationary state. Second, sufficient heterogeneity leads to the global saddle-path stability and the uniqueness of a given stationary state and the global uniqueness of equilibrium.
\end{abstract}

Keywords: Heterogeneity; Increasing Returns to Scale; Indeterminacy; Stability; Uniqueness.

JEL classification: $\mathrm{E} 1 ; \mathrm{O} 1$.

${ }^{1}$ Herrendorf, Departamento de Economía, Universidad Carlos III de Madrid; University of Warwick \& CEPR. E-mail: herrendo@eco.uc3m.es

2 Valentinyi, Department of Economics, University of Southampton \& CEPR. E-mail: av2@soton.ac.uk

${ }^{3}$ Waldmann, Department of Economics, Universitá di Chieti. E-mail: waldmann@datacomm.iue.it

\footnotetext{
* Forthcoming: the Review of Economic Studies. We are especially indebted to Martin Cripps for many clarifying discussions. For comments and suggestions, we moreover thank James Costain, Oded Galor, Takashi Kamihigashi, In Ho Lee, Andy Mountford, Edward C. Prescott, Manuel Santos, Hyun Song Shin, Daniel Tsiddon, two anonymous referees, and the audiences at presentations at Carlos III, Catholica in Milano, Dartmouth, Essex, Exeter, LSE, Michigan State, Minnesota, Federal Reserve Bank of Minneapolis, Munich, Northwestern, Pompeu Fabra, Southampton, Tilburg, Warwick, the Royal Economic Society Conference 1997, CEPR`s Summer Symposium in Macro 1998, the European Summer Meeting of the Econometric Society 1998, the Midwest Macro Conference in Bloomington 1998, the American Winter Meeting of the Econometric Society 1999, and the SED Conference 1999. Furthermore, Herrendorf thanks the Department of Economics at the Unviversity of Minnesota for its hospitality during 1998/99 when part of this paper was written, Valentinyi acknowledges financial support from the ACE Programme of the Commision of the European Communities 92-0200-S, and Waldmann thanks IGIER at Universitá Bocconi to which he was affiliated between 1996 and 1998.
} 


\section{Introduction}

The uniqueness of equilibrium is crucial in economic analysis. If multiple equilibria exist then the concept of rational expectations equilibrium becomes inconsistent unless an equilibrium selection mechanism can be identified by which non-economic factors, such as cultural conventions or institutional constraints, coordinate individual beliefs and thereby select an equilibrium. Since not much is known about this coordination process, models with multiple equilibria typically do not lead to sharp predictions. Moreover, when there are multiple equilibria, the outcome of comparative static exercises depends crucially on the way in which beliefs are coordinated. This problem becomes worse when there is indeterminacy of equilibrium. ${ }^{1}$ In this case, it is impossible to conduct comparative static exercises at all. For these reasons, it is an important task to identify conditions under which the equilibrium is determinate and conditions under which it is unique.

This paper is about the determinacy and uniqueness of equilibrium when there are economies of scale that are external to the individual decision makers. Economies of scale lead to a strategic complementarity, implying that beliefs can be self-fulfilling and the equilibrium can be non-unique [Cooper and John (1988)]. More specifically, when all individuals believe that all other individuals will undertake the activity that is subject to increasing returns, they may find it optimal to undertake that activity and thereby indeed increase its returns. ${ }^{2}$

We argue that the likelihood of non-unique equilibrium is overstated by most that literature. The reason is that it employs dynamic general equilibrium models with representative agents and restricts attention to symmetric equilibria. ${ }^{3}$ By construction of these models,

\footnotetext{
${ }^{1}$ Following Kehoe and Levine (1985) or Farmer (1993), we call the equlibrium indeterminate if it is locally non-isolated, that is, in each neighborhood there is another equilbirium.

${ }^{2}$ The implications of this phenomenon were studied by several authors in models with infinitely lived agents. For example, Benhabib and Farmer (1996) and Kiyotaki (1988) demonstrate that random fluctuations in self-fulfilling beliefs can generate business cycles, while Boldrin and Rustichini (1994) and Pelloni and Waldmann (1998) show that they can affect economic growth.

${ }^{3}$ Using such a model, Benhabib and Farmer (1996) e.g. demonstrate that indeterminacy is possible for mild increasing returns of an order of magnitude consistent with the empirical findings of Norrbin (1993)
} 
all individuals are identical, hold the same beliefs and act collectively, implying that the whole population can switch from one equilibrium to another one. This is crucial in the presence of increasing returns. There are at least two reasons why such collective switches may not occur. First, individuals may not be able to coordinate, for example because they face a free-riding problem or because they have heterogeneous information. ${ }^{4}$ The present paper points out why collective switches may not occur even when coordination is not an issue: when agents are heterogeneous with respect to their physical characteristics, not sufficiently many of them may find it optimal to act upon a change of beliefs. ${ }^{5}$

We study the role of heterogeneity in a version of a model developed by Matsuyama (1991). There are two sectors and increasing returns to scale. Individuals are differently productive and choose the sector in which they will work. Matsuyama found that there can be multiple equilibria, one of which can even be indeterminate. Our main contribution is to show that whether this is the case depends critically on the distribution of individual productivity. More precisely, starting from an arbitrary interior stationary state and an arbitrary distribution of individual productivity, we define a change in heterogeneity as a spread of that distribution and restrict attention to a class of spreads that leave the chosen interior stationary state invariant. We derive three results. First, sufficient homogeneity gives rise to multiple stationary states and to the indeterminacy and instability of the chosen stationary state. ${ }^{6}$ Second, for intermediate ranges of heterogeneity there are still multiple stationary states but the equilibrium close to the chosen stationary state becomes determinate although not necessarily unique. Third, sufficient heterogeneity ensures that

and Basu and Fernald (1997). Note, however, that this may no longer be true once one accounts for investment adjustment costs [Kim (1997)].

${ }^{4}$ In closely related but independent work, the first point is developed by Adsera and Ray (1998) and the second one by Karp (1999), who builds on an idea of Morris and Shin (1998).

${ }^{5}$ Recent evidence collected by Davis et al. (1996), for example, suggests that there is considerable heterogeneity with respect to job creation and destruction across U.S. manufacturing plants. Moreover, the results of Basu and Fernald (1997) indicate that heterogeneity across firms and industries with respect to the degree of increasing returns cannot be neglected.

${ }^{6}$ Note that each stationary state is an equilibrium if the initial conditions are the stationary state values of the relevant variables. 
the chosen stationary state becomes unique and globally saddle-path stable and that the equilibrium is unique everywhere. In other words, the occurrence of multiplicity and indeterminacy in our model requires that there is sufficient homogeneity across agents.

\section{Economic Environment}

We use a version of Matsuyama (1991) continuous time overlapping generations economy, in which at any point in time a continuum of measure one of individuals is alive. Matsuyama's model is well suited for our purposes because individuals are different with respect to their productivity. Moreover, the economy is small and open, there are no barriers to trade, and there are perfect capital and insurance markets, so individuals can freely borrow and lend at the given world real interest rate $r>0$ to smooth their consumption. This suppresses general equilibrium effects on prices and allows us to focus on the role of heterogeneity.

\subsection{Technology}

There are two sectors, called agriculture and manufacturing, which produce different goods. We assume that their relative world market price is constant and normalize it to one. All individuals are taken to be equally productive when they work in agriculture, and their endowment of labor services is normalized to one. The technology in agriculture transforms one unit of labor services into one unit of the output. In contrast, individuals are differently productive in manufacturing. In particular, there are different types $i \in \mathbb{R}$, which are distributed across the population according to a cumulative distribution function $G(\cdot)$.

Assumption $1 G(\cdot): \mathbb{R} \rightarrow[0,1]$ is exogenously given, time invariant, has a finite mean, and a continuous and positive density function $g(\cdot)$.

Note that the inverse of $G(\cdot)$ exists, is continuously differentiable, and maps $\mathbb{R}$ onto $[0,1]$. We denote it by $G^{-1}(\cdot)$. To give the different types an economic meaning, we assign the 
endowment of $e(i)$ units of labor services in manufacturing to type- $i$ individuals.

Assumption $2 e(\cdot): \mathbb{R} \rightarrow \mathbb{R}_{+}$is continuously differentiable with $e^{\prime}(i)<0 \forall i \in \mathbb{R}$ and has the range $[\underline{e}, \bar{e}] \subset(0, \infty)$.

This implies that $\lim _{i \rightarrow-\infty} e(i)=\bar{e}$ and $\lim _{i \rightarrow \infty} e(i)=\underline{e}$. To avoid confusion, it should be stressed that a lower $i$ is associated with a higher productivity. ${ }^{7}$ The technology in manufacturing has economies of scale due to an agglomeration externality that is external to each individual working there. In particular, individual output in manufacturing is assumed to be $a(n) e(i)$, where $n(t)$ is the fraction of the population in manufacturing at $t$.

Assumption $3 a(\cdot):[0,1] \rightarrow \mathbb{R}_{+}$is continuously differentiable with $a^{\prime}(n)>0 \forall n \in[0,1]$ and has the range $[\underline{a}, \bar{a}] \subset(0, \infty) .^{8}$

\subsection{Individuals' Problem}

There is a constant death rate $p>0$. If an individual dies it is replaced by a newborn of the same type. By the law of large numbers, the distribution of types then remains invariant and there is no aggregate uncertainty. ${ }^{9}$ At the beginning of its life, each individual makes an irreversible decision about the sector in which it will work. We assume that she chooses the sector with the larger expected present discounted lifetime income. ${ }^{10}$ The relevant information for the newborns' decisions at time $t$ is the future path of $n,\{n(s)\}_{s=t}^{\infty}$. Taking it as given and denoting the individual discount rate by $\rho, \rho \in(0, \infty)$, the formal condition

\footnotetext{
${ }^{7}$ Note that it might seem more natural to assume that $e^{\prime}(\cdot)>0$ and also to index the types directly by their endowments of labor services. Our choices will turn out to be convenient below but do not affect our results.

${ }^{8}$ This implies that $a(0)=a$ and $a(1)=\bar{a}$.

${ }^{9} \mathrm{Judd}(1985)$ shows that always there exists a measure such that the law of large number holds.

${ }^{10}$ Since there are perfect capital and insurance markets, individuals can freely borrow, and so this could easily be derived from a standard intertemporal maximization problem, given the choice of sector.
} 
that individuals of type $i$ born at time $t$ choose to work in manufacturing is

$$
E_{t}\left(\int_{t}^{\infty} \exp (-\rho(s-t)) d s\right) \leq E_{t}\left(\int_{t}^{\infty} a(n(s)) e(i) \exp (-\rho(s-t)) d s\right)
$$

where $E_{t}(\cdot)$ denotes the individual expectation at $t$. Given that the death rate is $p$, the probability that an individual born at time $t$ lives at least until time $s>t$ equals $\exp (-p(s-t))$. Using this $(1)$ can be rewritten to

$$
\frac{1}{e(i)} \leq V(t) \equiv(\rho+p) \int_{t}^{\infty} a(n(s)) \exp (-(\rho+p)(s-t)) d s
$$

where $V(t)$ represents the annuity value of the output stream that one unit of labor services produces in manufacturing. Note that $V(t) \in[\underline{a}, \bar{a}]$.

Since the distribution of types is over $i$, we want to rewrite inequality (2) as a condition on $i$. To this end, we need to extend the definition of $e^{-1}(\cdot)$, the inverse of $e(\cdot)$, so as to allow for the possibility that $1 / V(t)$ is not in the range of $e(\cdot)$ :

$$
e^{-1}(1 / V(t)) \equiv\left\{\begin{array}{lll}
-\infty & \text { if } & V(t)<1 / \bar{e} \\
\infty & \text { if } & V(t)>1 / \underline{e}
\end{array}\right.
$$

Since $e(\cdot)$ was assumed to be monotonically decreasing, (2) implies that all newborns of type $i \leq e^{-1}(1 / V(t))$ choose manufacturing, and so the fraction of newborns choosing manufacturing at time $t$ is given by $F(V(t)) \equiv G\left(e^{-1}(1 / V(t))\right)$.

\section{Competitive Equilibrium}

Definition 1 (Competitive Equilibrium) $A$ competitive equilibrium is an initial $\bar{n}_{0}$, paths $\{\hat{n}(t), \hat{V}(t)\}_{t=0}^{\infty}$ with $\hat{n}(0)=\bar{n}_{0}$, and individual choices of sector such that (i) at any point in time $t$, given $\hat{V}(t)$ each newborn's choice of sector maximizes her expected lifetime 
income, that is, (2) holds; (ii) the paths $\{\hat{n}(t), \hat{V}(t)\}_{t=0}^{\infty}$ are consistent with the newborns' career decisions, that is, $\hat{V}(t)$ is as in (2) and $\hat{n}(t)$ satisfies

$$
\hat{n}(t)=\hat{n}(0)+p \int_{0}^{t}[F(\hat{V}(s))-\hat{n}(s)] d s
$$

In words, the consistency requirements (2) and (4) say that the newborns' decisions generate a path for $\hat{n}(t)$ that is consistent with the path of $\hat{V}(t)$ that the newborns take as given when they made their decisions. In particular, equation (4) says that at each point in time the share of individuals working in manufacturing equals the initial share, $\hat{n}(0)$, plus the mass of individuals that entered manufacturing between time 0 and $t$, i.e. $p \int_{0}^{t} F(\hat{V}(s)) d s$, minus the mass of individuals that worked in manufacturing and died between time 0 and $t$, i.e. $p \int_{0}^{t} \hat{n}(s) d s$.

The equilibrium dynamics of our economy are fully characterized by the laws of motion for $V$ and $n$. Taking the derivatives of (2) and (4) with respect to time, we get the following two differential equations: ${ }^{11}$

$$
\begin{aligned}
& \dot{V}=(\rho+p)[V-a(n)], \\
& \dot{n}=p[F(V)-n] .
\end{aligned}
$$

Since $(n, V)$ remain in the compact set $[0,1] \times[\underline{a}, \bar{a}] \subset \mathbb{R}^{2}$, the system (5) has a unique solution for each pair $\left(\bar{n}_{0}, \bar{V}_{0}\right)$ of initial values; see Theorems 1.0.1 and 1.0.3 in Guckenheimer and Holmes (1983). To avoid confusion it should be stressed that many of these solutions violate the equilibrium conditions, so we still need to show that for each $\bar{n}_{0}$ an equilibrium exists.

A stationary state of the economy is a pair $\left(n^{*}, V^{*}\right)$ such that all variables are constant over time $(\dot{V}=\dot{n}=0)$. Since we are in a small open economy, consumption is only constant in stationary state if the world interest rate, $r$, equals the effective discount rate, $\rho+p$.

\footnotetext{
${ }^{11}$ In what follows we will drop the time index whenever this is unlikely to cause confusion.
} 
Figure 1: The Dynamics Under Assumptions 5(a) and 5(b)

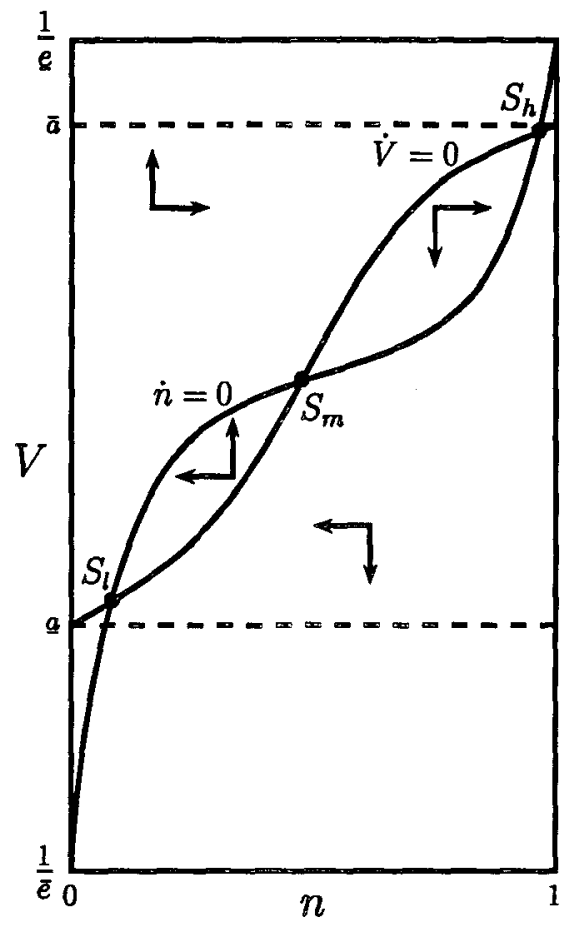

(a)

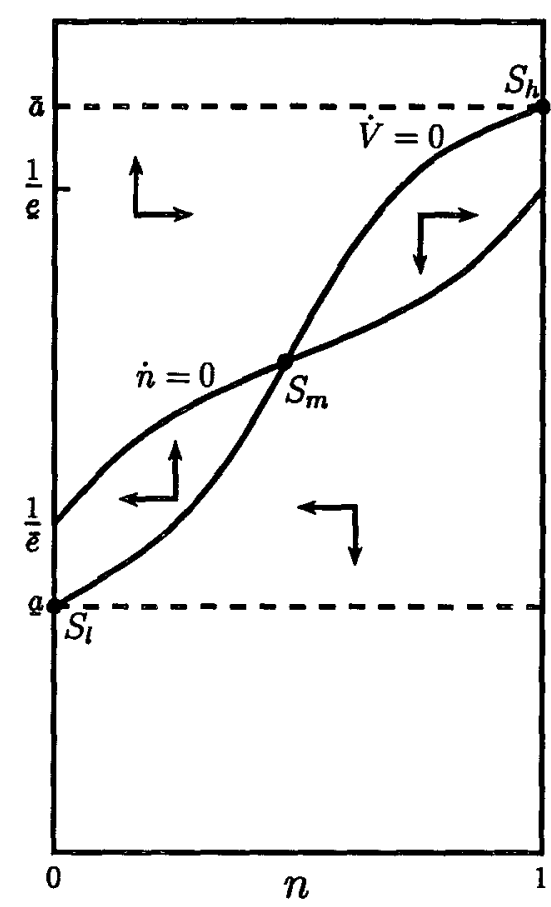

(b)

Assumption $4 r=\rho+p$.

Using (5), the conditions for a stationary state are found to be $V=a(n)$ and $V=F^{-1}(n)$. To ensure that an interior stationary state exists, we make

Assumption 5 Either $(a)[\underline{a}, \bar{a}] \subset[1 / \bar{e}, 1 / \underline{e}]$ or $(b)[1 / \bar{e}, 1 / \underline{e}] \subset[\underline{a}, \bar{a}]$.

As Figure 1 illustrates, Assumption 5(a) implies that some newborns are so productive (unproductive) in manufacturing that they (do not) choose it even if nobody (everybody) works there. In other words, Assumption 5(a) excludes stationary states at the corners. Similarly, Assumption 5(b) implies that if everybody works in agriculture (manufacturing) then all newborns choose agriculture (manufacturing). In other words, Assumption 5(b) ensures that both corners are stationary states, that is, $(0, \underline{a})$ and $(1, \bar{a})$ are stationary states. Moreover, given that all relevant functions are continuous, Assumption 5 ensures that an interior stationary state exists. Generically there is an odd number of stationary 
states. While each stationary state $\left(n^{*}, V^{*}\right)$ is an equilibrium for the initial condition $\bar{n}_{0}=n^{*}$, Assumptions $1-5$ ensure the existence of an equilibrium $\{\hat{n}(t), \hat{V}(t)\}_{t=0}^{\infty}$ with $\hat{n}(0)=\bar{n}_{0}$ for each $\bar{n}_{0} \in(0,1) .{ }^{12}$

Since a newborn's career decision depends on how many individuals will work in manufacturing in the future, the model has a strategic complementarity. Changes in individual beliefs about future decisions of other individuals can then be self-fulfilling and lead to the occurrence of multiple equilibria. It is important to distinguish the multiplicity of stationary state from the multiplicity of equilibrium. For example, in Figure 2 the economy has multiple stationary states in both cases, but in case (i) the equilibrium is unique for any initial $\bar{n}_{0}$, whereas in case (ii) there are initial $\bar{n}_{0}$ sufficiently close to the middle stationary state for which it is not unique. Moreover, in case (ii) the equilibrium is indeterminate (i.e. not isolated) when the economy is in the middle stationary state.

Definition 2 (Determinacy and Uniqueness) Let $\{\hat{n}(t), \hat{V}(t)\}_{t=0}^{\infty}$ be a competitive equilibrium with $\hat{n}(0)=\bar{n}_{0}$. (i) $\{\hat{n}(t), \hat{V}(t)\}_{t=0}^{\infty}$ is unique at $\hat{n}(t)$ if $\hat{V}(t)$ is the only $V \in$ $[\underline{a}, \bar{a}]$ for which $(\hat{n}(t), V)$ is on an equilibrium path. (ii) $\{\hat{n}(t), \hat{V}(t)\}_{t=0}^{\infty}$ is determinate at $\hat{n}(t)$ if it is locally unique, that is, there exists an $\varepsilon>0$ such that $\hat{V}(t)$ is the only $V \in(\hat{V}(t)-\varepsilon, \hat{V}(t)+\varepsilon)$ for which $(\hat{n}(t), V)$ is on an equilibrium path.

Note that determinacy (multiplicity of equilibrium) is a necessary but not a sufficient condition for the uniqueness of equilibrium (indeterminacy).

\footnotetext{
${ }^{12}$ This follows from the facts that (i) the stationary state with the smallest $n^{*}$ must be saddle-path stable, the next one (as $n^{*}$ increases) must be unstable, followed by a saddle-path stable stationary state and so forth; (ii) for each saddle-path stable stationary state the stable manifold is differentiable in that stationary state and so there are two paths coming from different directions; (iii) since the solution to (5) is unique, different manifolds can cross only in a stationary state. Hence, there is at least one $\hat{V}(0)$ such that $\left(\bar{n}_{0}, \hat{V}(0)\right)$ lie on a stable manifold. The unique solution to the dynamical system (5) with initial values $\left(\bar{n}_{0}, V(0)\right)$ then is an equilibrium path with initial $\bar{n}_{0}$.
} 
Figure 2: Possible Equilibrium Dynamics Under Assumption 5(a)

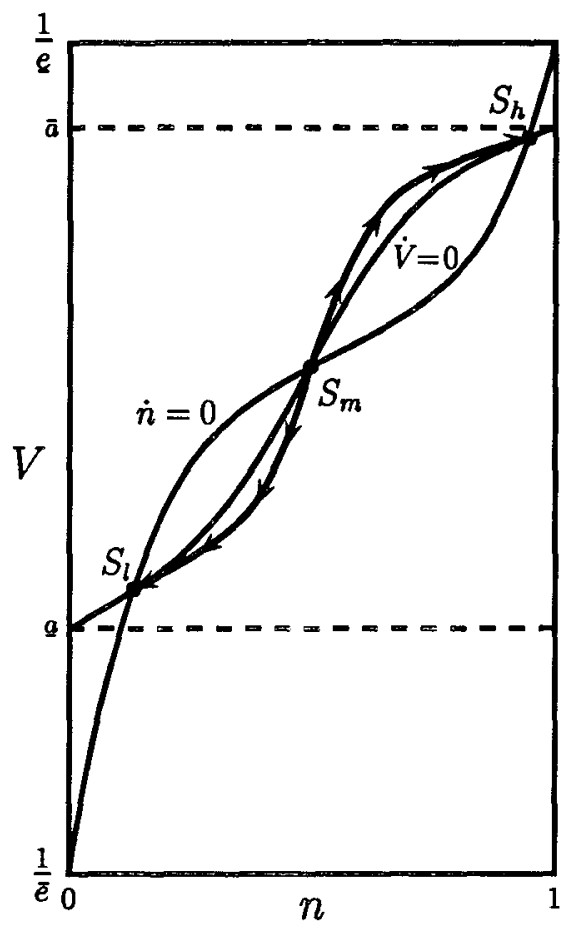

(i)

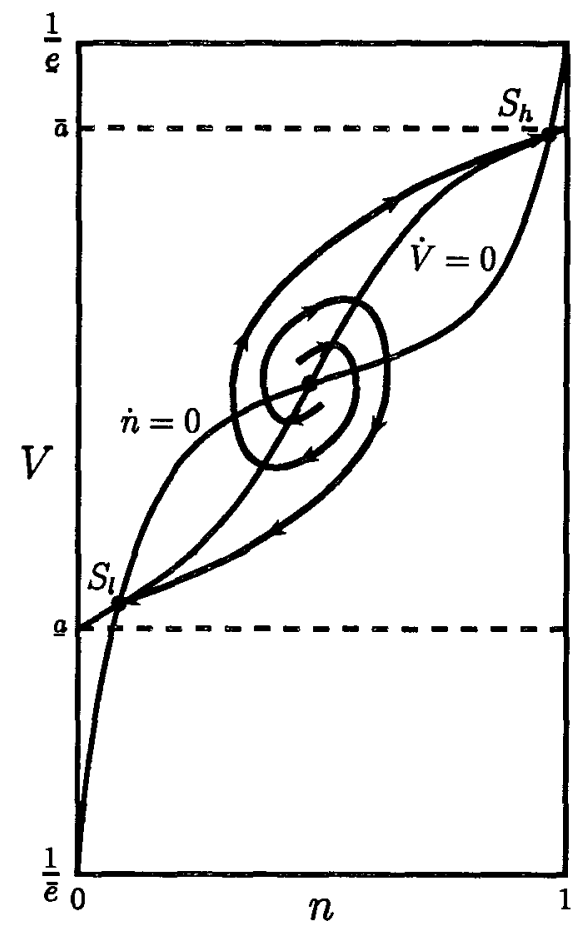

(ii)

\section{Parametrization of Heterogeneity}

We borrow from Rothschild and Stiglitz (1970) and restrict our attention to those changes in heterogeneity that correspond to spreads of the original distribution function. ${ }^{13}$

Definition 3 (Increase in Heterogeneity) Let $G_{1}(\cdot)$ and $G_{2}(\cdot)$ be two distribution functions with support $\mathbb{R}$. There is more heterogeneity under $G_{2}(\cdot)$ than under $G_{1}(\cdot)$ if $G_{2}(\cdot)$ is a spread of $G_{1}(\cdot)$, that is, there is a single crossing point $i_{c}$ such that $G_{1}(i)<G_{2}(i)$ for $i<i_{c} ; G_{1}\left(i_{c}\right)=G_{2}\left(i_{c}\right) ; G_{1}(i)>G_{2}(i)$ for $i>i_{c}$. If $G_{2}(\cdot)$ is a spread of $G_{1}(\cdot)$ then $G_{1}(\cdot)$ is a shrink of $G_{2}(\cdot)$ and there is more homogeneity under $G_{1}(\cdot)$ than under $G_{2}(\cdot)$.

In general, a distribution and its spread can have more than one crossing point. We consider only spreads and shrinks with a single crossing point because this allows us to

\footnotetext{
${ }^{13}$ Note that we do not require the spreads to be mean preserving. If we did then our definition would be equivalent to first-order stochastic dominance.
} 
Figure 3: Construction of a Spread

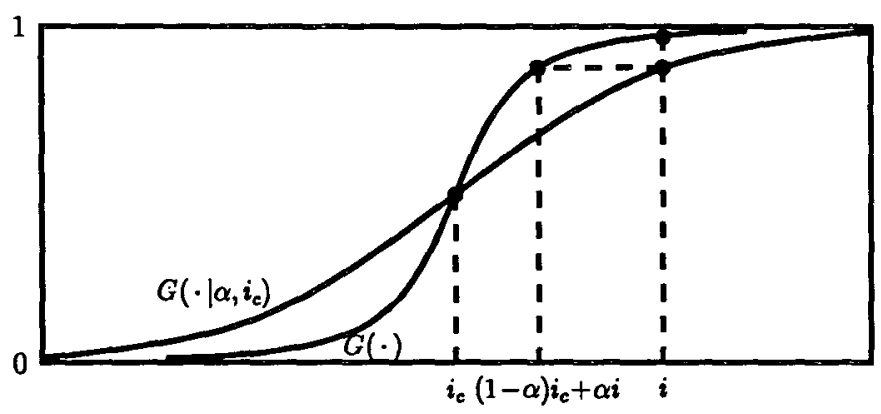

parameterize them in the following parsimonious way:

$$
G\left(i \mid \alpha, i_{c}\right) \equiv G\left(\alpha i+(1-\alpha) i_{c}\right), \quad \alpha \in(0, \infty)
$$

The idea underlying this construction is illustrated by Figure 3. It is straightforward to prove if $\alpha \in(0,1)$, then $G\left(\cdot \mid \alpha, i_{c}\right)$ is a spread of $G(\cdot)$ and if $\alpha \in(1, \infty)$, then $G\left(\cdot \mid \alpha, i_{c}\right)$ is a shrink of $G(\cdot)$. Furthermore, the parameter $\alpha$ measures the change in heterogeneity relative to the original distribution $G(\cdot)$ : the lower is $\alpha$ the more heterogeneous is the population. Our strategy for investigating the effects of changes in heterogeneity will be to pick a specific interior stationary state, $\left(n^{*}, V^{*}\right)$, of the economy with $i$ distributed according $G(\cdot)$ and then to change heterogeneity in such a way that $\left(n^{*}, V^{*}\right)$ remains a stationary state for all $\alpha \in(0, \infty)$ when $i$ is distributed according to $G\left(\cdot \mid \alpha, i_{c}\right)$. This requires us to choose $i_{c}=e^{-1}\left(1 / V^{*}\right) .^{14}$ For future reference it is useful to introduce $F\left(V \mid \alpha, V^{*}\right) \equiv G\left(e^{-1}(1 / V) \mid \alpha, e^{-1}\left(1 / V^{*}\right)\right)$.

${ }^{14}$ To see this, note that (i) the $\dot{V}=0$ locus does not depend on $\alpha$; (ii) the $\dot{n}=0$ locus remains invariant at $n^{*}$ if and only if $G\left(e^{-1}\left(1 / V^{*}\right) \mid \alpha, i_{c}\right)=n^{*}=G\left(e^{-1}\left(1 / V^{*}\right)\right)$; (iii) $i_{c}$ is the only crossing point of the two spreads. 


\section{Ruling out Multiplicity and Indeterminacy: \\ the Role of Heterogeneity}

Here, we analytically characterize the role of heterogeneity. The first Proposition focuses on the local properties of the chosen stationary state, $\left(n^{*}, V^{*}\right)$, and the equilibrium.

Proposition 1 (Saddle-Path Stability and Determinacy) Suppose Assumptions 15 are satisfied, $\left(n^{*}, V^{*}\right)$ is an interior stationary state of the economy when $i$ is distributed according to $G(\cdot)$, and

$$
\begin{aligned}
& \bar{\alpha}_{1} \equiv \frac{-e^{\prime}\left(e^{-1}\left(1 / V^{*}\right)\right) V^{* 2}}{a^{\prime}\left(n^{*}\right) g\left(e^{-1}\left(1 / V^{*}\right)\right)} \\
& \bar{\alpha}_{2} \equiv\left[1+\frac{(r-p)^{2}}{4 p r}\right] \bar{\alpha}_{1}
\end{aligned}
$$

(i) Stability properties of $\left(n^{*}, V^{*}\right)$ when $i$ is distributed according to $G\left(\cdot \mid \alpha, e^{-1}\left(1 / V^{*}\right)\right)$ : if $\alpha \in\left(0, \bar{\alpha}_{1}\right)$ then $\left(n^{*}, V^{*}\right)$ is locally saddle-path stable; if $\alpha \in\left(\bar{\alpha}_{1}, \infty\right)$ then $\left(n^{*}, V^{*}\right)$ is unstable. ${ }^{15}$

(ii) Determinacy properties of equilibrium close to $\left(n^{*}, V^{*}\right)$ when $i$ is distributed according to $G\left(\cdot \mid \alpha, e^{-1}\left(1 / V^{*}\right)\right)$ : if $\alpha \in\left(0, \bar{\alpha}_{2}\right)$ then $\left(n^{*}, V^{*}\right)$ and all equilibria with $(\hat{n}(t), \hat{V}(t))$ sufficiently close to $\left(n^{*}, V^{*}\right)$ are determinate at these $\hat{n}(t) ;$ if $\alpha \in\left(\bar{\alpha}_{2}, \infty\right)$ then $\left(n^{*}, V^{*}\right)$ is indeterminate at $n^{*}$.

The results of Proposition 1 are illustrated by Figure 4. To develop some intuition, note that the slope of the $\dot{n}=0$ locus at the chosen interior stationary state is given by the product of some positive constant and $1 / \alpha$. Thus, small $\alpha$ 's make the $\dot{n}=0$ locus at $\left(n^{*}, V^{*}\right)$ steeper than the $\dot{V}=0$ locus, that is, $\left(n^{*}, V^{*}\right)$ locally saddle-path stable. This

\footnotetext{
${ }^{15}$ The potential stabilizing effect of heterogeneity is not unknown in economics. Grandmont (1992), for instance, shows that heterogeneous preferences can ensure that the aggregate demand curve is well behaved. To our knowledge, however, it has not previously been investigated how heterogeneity affects the stability properties in a dynamic model.
} 
Figure 4: The Role of Heterogeneity

Chosen Interior

Stationary State $\left(n^{*}, V^{*}\right)$

Equilibrium

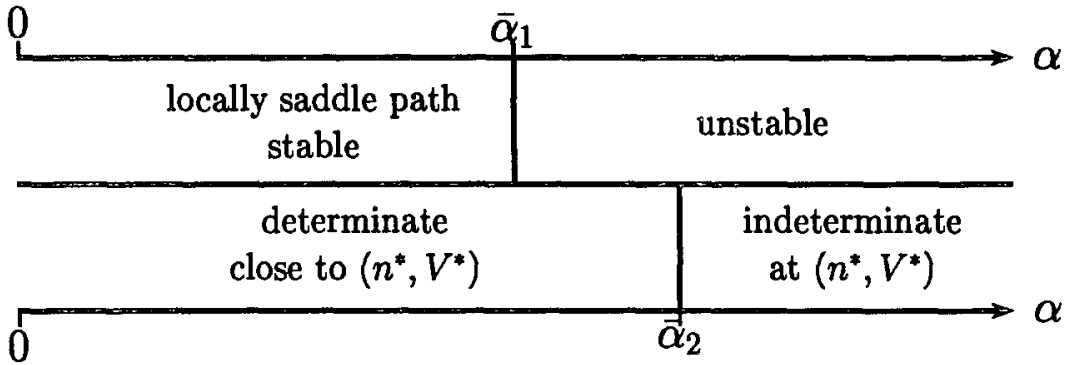

also implies the determinacy of equilibrium close to $\left(n^{*}, V^{*}\right)$. The opposite statement holds true for large $\alpha$ 's. In economic terms, changing $\alpha$ such that $\left(n^{*}, V^{*}\right)$ remains a stationary state changes the mass of individuals with endowments of labor services in manufacturing close to $1 / V^{*}$. For large $\alpha$, that masss is relatively large. If returns are sufficiently strongly increasing, beliefs can then be self-fulfilling: if all individuals with endowments of labor services close to $1 / V^{*}$ believe that all the others with similar endowments are going to enter manufacturing, that will increase output in manufacturing by enough to make it attractive for all them to enter. Small changes in $V$ can then change the decisions of many newborns, implying that the chosen stationary state is unstable and the stationary state equilibrium indeterminate. The opposite statements apply for small $\alpha$ 's.

It is interesting to observe from (7a) that if $\left(n^{*}, V^{*}\right)$ is initially locally saddle-path stable (unstable) then increasing heterogeneity (homogeneity) does not change this. Moreover, (7a) and (7b) show that if returns are strongly increasing (i.e. $a^{\prime}(\cdot)$ is relatively large) or if individuals discount the future at a low rate (i.e. $r$ is small) then the entry of fewer individuals into manufacturing can make beliefs self-fulfilling. Moreover, if the death rate $p$ is relatively large, a relatively large number of newborns make decisions at any point in time and the previous two effects get amplified. In all cases, more heterogeneity is required (i.e. $\bar{\alpha}_{2}$ falls) to rule out the possibility of self-fulfilling beliefs and bring about determinacy. ${ }^{16}$

\footnotetext{
${ }^{16}$ Two remarks about the related literature are at order. First, our result that for any rate of time preference $\rho=r-p$ enough heterogeneity makes equilibrium determinate complements what Matsuyama (1991)
} 
Figure 5: The Role of Heterogeneity When All Stationary States are Interior

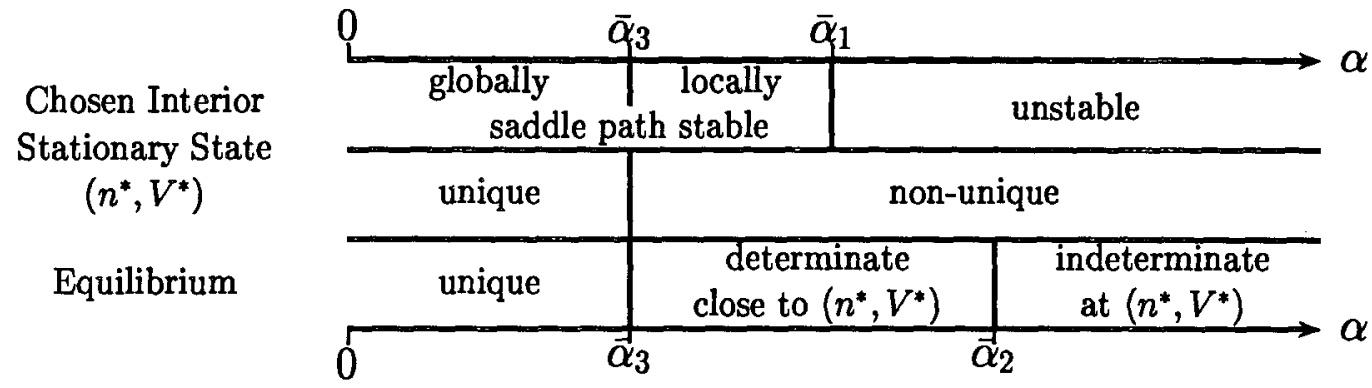

We now show that under Assumption 5(a) we can derive some global results.

\section{Proposition 2 (Uniqueness and Global Saddle-path Stability) Suppose Assump-} tions 1-5 (a) are satisfied and $\left(n^{*}, V^{*}\right)$ is an interior stationary state of the economy when $i$ is distributed according to $G(\cdot)$. There exists a unique $\bar{\alpha}_{3} \in\left(0, \bar{\alpha}_{1}\right]$ with $\bar{\alpha}_{1}$ defined in (7a) such that:

(i) if $\alpha \in\left(0, \bar{\alpha}_{3}\right)$ and $i$ is distributed according to $G\left(\cdot \mid \alpha, e^{-1}\left(1 / V^{*}\right)\right)$ then $\left(n^{*}, V^{*}\right)$ is the unique stationary state, $\left(n^{*}, V^{*}\right)$ is globally saddle-path stable, and the equilibrium is unique for all initial $\bar{n}_{0} \in[0,1]$;

(ii) if $\alpha \in\left(\bar{\alpha}_{3}, \infty\right)$ and $i$ is distributed according to $G\left(\cdot \mid \alpha, e^{-1}\left(1 / V^{*}\right)\right)$ then $\left(n^{*}, V^{*}\right)$ is one of multiple stationary states.

Recalling that $\bar{\alpha}_{3} \leq \bar{\alpha}_{1}<\bar{\alpha}_{2}$, we can summarize the results when Assumption 5(a) holds by Figure $5{ }^{17}$ Intuitively, the global results of Proposition 2 can be understood as follows. Assumption 5(a) ensures that individual productivity characteristics are sufficiently heterogeneous so that there are individuals with $\underline{a}>1 / e(i)$ and individuals with $\bar{a}<1 / e(i)$.

found, notably that for any amount of heterogeneity there is a rate of time preference such that equilibrium is indeterminate. Second, in independent work, Schmutzler (1998) also points out that heterogeneity tends to work in the opposite direction from increasing returns. Since his models always has a unique equilibrium, he does not, however, study the effects of heterogeneity on the determinacy of equilibrium.

${ }^{17}$ Note that one might think that a sufficient condition for the uniqueness of equilibrium is that both roots are real. That this is incorrect is illustrated by Figure Vb on page 646 in Matsuyama (1991). 
Figure 6: Heterogeneity and the Uniqueness of Equilibrium under Assumption 5(a)

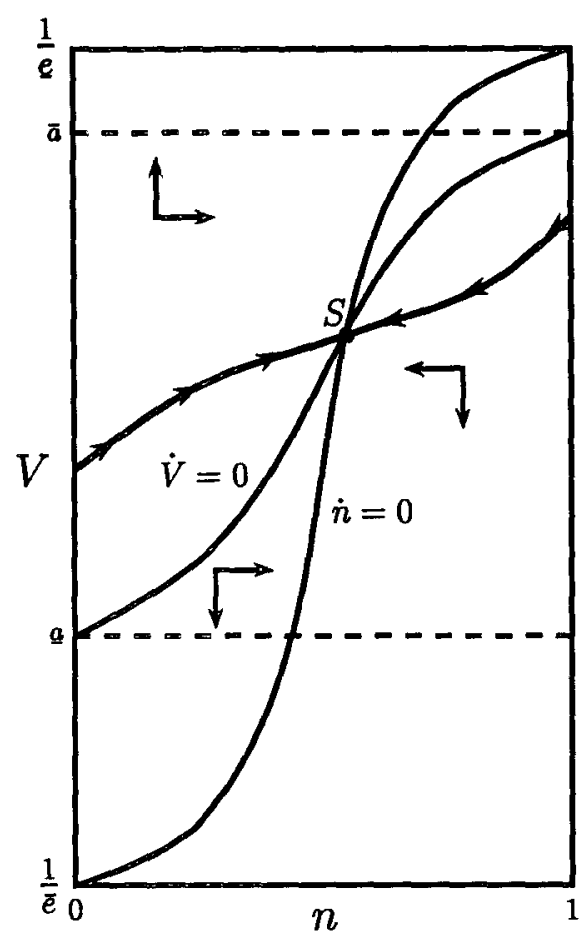

Regardless of what the other agents do, the former individuals always choose manufacturing whereas the latter ones always choose agriculture. Since increasing heterogeneity spreads out individual characteristics and so increases the masses of both types, enough heterogeneity makes the chosen stationary state unique and saddle-path stable and the equilibrium unique. In graphical terms, enough heterogeneity makes the $\dot{n}=0$ curve so steep in the relevant area that it intersects exactly once with the $\dot{V}=0$ curve; see Figure 6. ${ }^{18}$

\footnotetext{
${ }^{18}$ In independent research, Frankel and Pauzner (1998) introduce a different form of heterogeneity into Matsuyama's model. In particular, they add persistent aggregate productivity shocks, which make the problem of identical types at different points in time different. They find that this form of heterogeneity can lead to the uniqueness of equilibrium too.
} 


\section{Conclusion}

This paper has studied the relation between the degree of heterogeneity in the population and the properties of equilibrium and of a chosen stationary state in Matsuyama's (1991) two-sector model with increasing returns to scale. We have found three results. First, sufficient homogeneity gives rise to multiple stationary states, to the instability of the chosen stationary state, and to the indeterminacy of equilibrium at that stationary state. Second, for intermediate ranges of heterogeneity there are still multiple stationary states but the equilibrium close to the chosen stationary state is determinate although not necessarily unique. Third, sufficient heterogeneity ensures that the chosen stationary state is unique and globally saddle-path stable and that the equilibrium is unique everywhere. These results suggest that homogeneity favors the occurrence of multiplicity and indeterminacy.

\section{Appendix}

\section{A Proof of Proposition 1}

We know from the Stable Manifold Theorem that if a stationary state is hyperbolic (i.e. has no eigenvalues with zero real part) then the stability properties of the linearized system close to that stationary state are the same as those of the non-linear one. ${ }^{19}$ So we replace $G(\cdot)$ by $G\left(\cdot \mid \alpha, e^{-1}\left(1 / V^{*}\right)\right)$ in equations (5) and linearize the resulting system of equations around the chosen interior stationary state $\left(n^{*}, V^{*}\right)$ :

$$
\left[\begin{array}{l}
\dot{V} \\
\dot{n}
\end{array}\right]=\left[\begin{array}{cc}
\rho+p & -(\rho+p) a^{\prime}\left(n^{*}\right) \\
-p \frac{\alpha g\left(e^{-1}\left(1 / V^{*}\right)\right)}{e^{\prime}\left(e^{-1}\left(1 / V^{*}\right)\right) V^{* 2}} & -p
\end{array}\right]\left[\begin{array}{l}
V-V^{*} \\
n-n^{*}
\end{array}\right] \text {. }
$$

\footnotetext{
${ }^{19}$ See Guckenheimer and Holmes (1983, page13).
} 
The determinant and the eigenvalues of the above matrix are

$$
\begin{aligned}
D(\alpha) & \equiv p(\rho+p)\left[\frac{\alpha g\left(e^{-1}\left(1 / V^{*}\right)\right) a^{\prime}\left(n^{*}\right)}{-e^{\prime}\left(e^{-1}\left(1 / V^{*}\right)\right) V^{* 2}}-1\right] \\
\lambda_{1,2} & =\frac{\rho \pm \sqrt{\rho^{2}-4 D(\alpha)}}{2}
\end{aligned}
$$

Since $\rho \in(0,1), \lambda_{1,2}$ have non-zero real parts and the Stable Manifold Theorem applies.

For all $\alpha \in\left(0, \bar{\alpha}_{1}\right)$ the determinant (A.2a) is negative and $\left(n^{*}, V^{*}\right)$ is locally saddle-path stable. The determinant is positive for all $\alpha \in\left(\bar{\alpha}_{1}, \infty\right)$. As (A.2b) shows it is impossible in our model that both eigenvalues have negative real part. Thus, they must then both be real and positive or be complex conjugates with a positive real part, implying $\left(n^{*}, V^{*}\right)$ is unstable.

To prove part (ii), note that (A.2a) and (A.2b) imply that the roots of (A.1) are real if and only if $\alpha \in\left(0, \bar{\alpha}_{2}\right)$. In this cas, there is an $\varepsilon>0$ such that any equilibrium which has $(\hat{n}(t), \hat{V}(t)) \in\left(n^{*}-\varepsilon, n^{*}+\varepsilon\right) \times\left(V^{*}-\varepsilon, V^{*}+\varepsilon\right)$ is determinate at these $\hat{n}(t)$. The roots are complex conjugates if and only if $\alpha \in\left(\bar{\alpha}_{2}, \infty\right)$. The manifold originating in $\left(n^{*}, V^{*}\right)$ then "spirals out of" $\left(n^{*}, V^{*}\right)$, which is illustrated by Figure 2. In this case, the chosen stationary state $\left(n^{*}, V^{*}\right)$ is an indeterminate equilibrium.

\section{B Proof of Proposition 2}

We start with part (i). First, we prove by contradiction that $\exists \alpha$ such that $\left(n^{*}, V^{*}\right)$ is unique. So, suppose that this is false. Then $\exists \alpha_{j} \in\left(0,1 / 2^{j}\right)$ for any $j \in \mathbb{N}$ such that the economy with $i$ distributed according to $G\left(\cdot \mid \alpha_{j}, e^{-1}\left(1 / V^{*}\right)\right)$ has two stationary states, $\left(n^{*}, V^{*}\right)$ and $\left(n^{* *}\left(\alpha_{j}\right), V^{* *}\left(\alpha_{j}\right)\right)$. Since all stationary states must satisfy $V=a(n)$ and $V=F^{-1}(n)$, we have $e^{-1}\left(1 / a\left(n^{*}\right)\right)=G^{-1}\left(n^{*}\right)$ and $e^{-1}\left(1 / a\left(n^{* *}\left(\alpha_{j}\right)\right)\right)=G^{-1}\left(n^{* *}\left(\alpha_{j}\right) \mid \alpha_{j}, e^{-1}\left(1 / V^{*}\right)\right)$, and 


$$
\begin{aligned}
& \mathrm{so}^{20} \\
& e^{-1}\left(1 / a\left(n^{* *}\left(\alpha_{j}\right)\right)\right)-e^{-1}\left(1 / a\left(n^{*}\right)\right)=G^{-1}\left(n^{* *}\left(\alpha_{j}\right) \mid \alpha_{j}, e^{-1}\left(1 / V^{*}\right)\right)-G^{-1}\left(n^{*} \mid \alpha_{j}, e^{-1}\left(1 / V^{*}\right)\right) .
\end{aligned}
$$

Furthermore, the construction of the spread given by equation (6) implies $^{21}$

$$
G^{-1}\left(n^{* *}\left(\alpha_{j}\right)\right)=\left(1-\alpha_{j}\right) G^{-1}\left(n^{*}\right)+\alpha_{j} G^{-1}\left(n^{* *}\left(\alpha_{j}\right) \mid \alpha_{j}, e^{-1}\left(1 / V^{*}\right)\right)
$$

Since $G^{-1}\left(n^{*} \mid \alpha_{j}, e^{-1}\left(1 / V^{*}\right)\right)=G^{-1}\left(n^{*}\right)$, this is equivalent to

$$
G^{-1}\left(n^{* *}\left(\alpha_{j}\right) \mid \alpha_{j}, e^{-1}\left(1 / V^{*}\right)\right)-G^{-1}\left(n^{*} \mid \alpha_{j}, e^{-1}\left(1 / V^{*}\right)\right)=\frac{G^{-1}\left(n^{* *}\left(\alpha_{j}\right)\right)-G^{-1}\left(n^{*}\right)}{\alpha_{j}} .
$$

Combining (B.1) and (B.3) and recalling that $\alpha_{j}>1 / 2^{j}$, this gives

$$
e^{-1}\left(1 / a\left(n^{* *}\left(\alpha_{j}\right)\right)\right)-e^{-1}\left(1 / a\left(n^{*}\right)\right) \geq 2^{j}\left[G^{-1}\left(n^{* *}\left(\alpha_{j}\right)\right)-G^{-1}\left(n^{*}\right)\right] \quad \forall j \in \mathbb{N} .
$$

(B.4) is contradicted and our proof by contradiction is completed if we can show that there is a $\kappa \in(0, \infty)$ such that for all $n_{1}, n_{2} \in[0,1]$ with $n_{1} \neq n_{2}$

$$
e^{-1}\left(1 / a\left(n_{1}\right)\right)-e^{-1}\left(1 / a\left(n_{2}\right)\right) \leq \kappa\left[G^{-1}\left(n_{1}\right)-G^{-1}\left(n_{2}\right)\right]
$$

To see this, note first that $e^{-1}(1 / a(\cdot))$ is continuously differentiable and monotonically increasing. Hence, for all $n_{1}, n_{2} \in[0,1] n_{1} \neq n_{2}$ we have

$$
\frac{e^{-1}\left(1 / a\left(n_{1}\right)\right)-e^{-1}\left(1 / a\left(n_{2}\right)\right)}{n_{1}-n_{2}} \leq \max _{n \in[0,1]} \frac{d e^{-1}(1 / a(n))}{d n}=\max _{n \in[0,1]} \frac{a^{\prime}(n)}{-e^{\prime}\left(e^{-1}(1 / a(n))\right)[a(n)]^{2}} .
$$

\footnotetext{
${ }^{20}$ Note that Assumption $5(\mathrm{a})$ implies $[1 / \bar{a}, 1 / a] \subset[e, \bar{e}]$ ensuring that $e^{-1}(1 / a(n))$ is well defined for all
$n \in[0,1]$.

${ }^{21}$ Figure 3 also illustrates this. $G^{-1}\left(n^{*}\right)$ and $G^{-1}\left(n^{* *}\left(\alpha_{j}\right) \mid \alpha_{j}, e^{-1}\left(1 / V^{*}\right)\right)$ correspond to $i_{c}$ and $i$ in the
figure, respectively.
} 
Since $G^{-1}(\cdot)$ is differentiable and $g(\cdot)$ is a positive and bounded density function, we also have that for all $n_{1}, n_{2} \in[0,1]$

$$
\frac{G^{-1}\left(n_{1}\right)-G^{-1}\left(n_{2}\right)}{n_{1}-n_{2}} \geq \min _{n \in[0,1]} \frac{d G^{-1}(n)}{d n}=\frac{1}{\max _{i \in \mathbb{R}} g(i)} \in(0, \infty) .
$$

(B.5) follows immediately by dividing (B.6) by (B.7) and setting

$$
\kappa \equiv \max _{n \in[0,1]} \frac{a^{\prime}(n)}{-e^{\prime}\left(e^{-1}(1 / a(n))\right)[a(n)]^{2}} \times \max _{i \in \mathbb{R}} g(i)
$$

and noticing that $\kappa \in(0, \infty)$. Since (B.4) and (B.5) contradict each other, this shows that there must exist an $\alpha$ for which the chosen stationary state $\left(n^{*}, V^{*}\right)$ is unique.

Next, we show that there is a $\bar{\alpha}_{3}$ such that for all $\alpha<\bar{\alpha}_{3}$ the chosen stationary state is unique and for all $\alpha>\bar{\alpha}_{3}$ it is not unique. From the previous step we know that the set of $\alpha$ 's for which the economy has multiple stationary states has the positive lower bound $1 / \kappa$. This implies that the infimum of that set exists and is positive. We call that infimum $\bar{\alpha}_{3}$. Since the slope of the $\dot{n}=0$ locus increases everywhere as $\alpha$ decreases and since the $\dot{V}=0$ locus does not depend on $\alpha$, we know that if the $\dot{V}=0$ and the $\dot{n}=0$ locus intersect more than once (exactly once) for some $\tilde{\alpha}$ then they interest more than once (exactly once) for all $\alpha>\tilde{\alpha}(\alpha<\tilde{\alpha})$. But this means that for all $\alpha \in\left(0, \bar{\alpha}_{3}\right)$ the chosen stationary state is unique, whereas for all $\alpha \in\left(\bar{\alpha}_{3}, \infty\right)$ it is not, which proves part (ii) of the proposition.

To show the saddle-path stability part of claim (i), observe that from (A.2a) it follows that the chosen stationary state $\left(n^{*}, V^{*}\right)$ is locally saddle-path stable iff

$$
a^{\prime}\left(n^{*}\right)<\left.\frac{d F^{-1}\left(n \mid \alpha, e^{-1}\left(1 / V^{*}\right)\right)}{d n}\right|_{n=n^{*}}
$$

The uniqueness of $\left(n^{*}, V^{*}\right)$ for $\alpha \in\left(0, \bar{\alpha}_{2}\right)$ implies that the $\dot{n}=0$ locus crosses the $\dot{V}=0$ locus exactly once. Therefore, as $\left(n^{*}, V^{*}\right)$ is a crossing point, it must be the only one. So, since $F^{-1}\left(0 \mid \alpha, e^{-1}\left(1 / V^{*}\right)\right)=1 / \bar{e}>\underline{a}=a(0)$, the $\dot{V}=0$ locus is flatter than the $\dot{n}=0$ 
locus in $\left(n^{*}, V^{*}\right)$. This is equivalent to (B.9).

To complete the proof of part (i), we still need to show that if the chosen stationary state is unique and locally saddle-path stable then it is globally saddle-path stable too. This can be seen in two steps. First, the saddle-path cannot start anywhere but at the boundary, the reason being that any other starting point would be another stationary state and thus contradict uniqueness. Second, the dynamics represented by the arrows on Figure 1b imply that any path starting at the boundary and reaching the $\dot{V}=0(\dot{n}=0)$ locus at some $V \neq V^{*}\left(n \neq n^{*}\right)$ can never reach $\left(n^{*}, V^{*}\right)$. Therefore, the chosen stationary state is globally saddle-path stable. Note that uniqueness and global saddle-path stability imply that for each $\bar{n}_{0} \in[0,1]$ there is a unique $\hat{V}(0)$ such that the unique solution to the system (5) with initial conditions $\left(\bar{n}_{0}, \hat{V}(0)\right)$ is a subset of the stable manifold. In other words, the equilibrium is unique if $\alpha \in\left(0, \bar{\alpha}_{2}\right)$.

Finally, we have not yet shown that $\bar{\alpha}_{3} \leq \bar{\alpha}_{1}$. This follows from Proposition 1 (which said that $\left(n^{*}, V^{*}\right)$ is unstable for all $\left.\alpha \in\left(\bar{\alpha}_{1}, \infty\right)\right)$ and the fact that $\left(n^{*}, V^{*}\right)$ is unique for all $\alpha \in\left(0, \bar{\alpha}_{2}\right)$. Since we have just seen that an unstable stationary cannot be unique, we must have $\bar{\alpha}_{3} \leq \bar{\alpha}_{1}$.

\section{References}

Adsera, A. and Ray, D. (1998). 'History and coordination failure.' Journal of Economic Growth, vol. 3, pp. 267-276.

Basu, S. and Fernald, J. G. (1997). 'Returns to scale in u.s. production: Estimates and implications.' Journal of Political Economy, vol. 105, pp. 249-283.

Benhabib, J. and Farmer, R. E. A. (1996). 'Indeterminancy and sector-specific externalities.' Journal of Monetary Economics, vol. 37, pp. 421-443. 
Boldrin, M. and Rustichini, A. (1994). 'Growth and indeterminacy in dynamic models with externalities.' Econometrica, vol. 62, pp. 323-342.

Cooper, R. and John, A. (1988). 'Coordinating coordination failures in keynesian models.' Quarterly Journal of Economics, vol. 103, pp. 441-463.

Davis, S. J. et al. (1996). Job Creation and Job Destruction. Cambridge, MA: MIT Press.

Farmer, R. E. A. (1993). The Macroeconomics of Self-Fulfilling Prophecies. Cambridge, MA: MIT Press.

Frankel, D. and Pauzner, A. (1998). 'Resolving indeterminacy in dynamic settings: the role of shocks.' Manuscript, Tel Aviv University.

Grandmont, J.-M. (1992). 'Transformation of commodity space, behavioral heterogeneity, and the aggregation problem.' Journal of Economic Theory, vol. 57, pp. 1-35.

Guckenheimer, J. and Holmes, P. (1983). Nonlinear Oscillations, Dynamical Systems, and Bifurcations of Vector Fields. New York: Springer Verlag.

Judd, K. L. (1985). 'The law of large numbers with a continuum of iid random variables.' Journal of Economic Theory, vol. 35, pp. 19-25.

Karp, L. (1999). 'History versus expectations with incomplete information.' Mimeo, University of California, Berkeley.

Kehoe, T. J. and Levine, D. K. (1985). 'Comparative statics and perfect foresight in infinite horizon economies.' Econometrica, vol. 53, pp. 433-453.

Kim, J. (1997). 'Indeterminacy and investment adjustment cost.' mimeo, University of Virginia.

Kiyotaki, N. (1988). 'Multiple expectational equilibria under monopolistic competition.' Quarterly Joural of Economics, vol. 105, pp. 695-713. 
Matsuyama, K. (1991). 'Increasing returns, industrialization, and indeterminacy of equilibrium.' Quarterly Journal of Economics, vol. 104, pp. 617-650.

Morris, S. and Shin, H. S. (1998). 'Unique equilibirum in a model of self-fulfilling currency attacks.' American Economic Review, vol. 88, pp. 587-597.

Norrbin, S. (1993). 'The relation between price and marginal cost in us industry: a contradiction.' Journal of Political Economy, vol. 101, pp. 1149-1164.

Pelloni, A. and Waldmann, R. (1998). 'Stability properties in a growth model.' Economics Letters, vol. 61, pp. 55-60.

Rothschild, M. and Stiglitz, J. E. (1970). 'Increasing risk: I. a definition.' Journal of Economic Theory, vol. 2, pp. 225-243.

Schmutzler, A. (1998) 'Changing places - the role of heterogeneity and externalities in cumulative processes.' International Journal of Industrial Organization, vol. 16, pp. 445-461. 